\title{
q-Power Quasi Binormal Operator
}

\begin{abstract}
Alaa Hussein Mohammed
Department of Mathematics, College of Education, University of Al-Qadisiyah, Diwaniya, Iraq e-mail: alaa.hussein@qu.edu.iq
\end{abstract}

\begin{abstract}
In this paper we introduce a new class of operators on Hilbert space called $q$-power quasi binormal operator. We study this operator and give some properties of it.
\end{abstract}

\section{Introduction}

Consider $B(H)$ be the algebra of all bounded linear operators on Hilbert space $H$. An operator $S$ is called normal if $S^{*} S=S S^{*}$. Quasi normal operator was introduced by Brown in 1953 [1]. In [3] Campbell introduced the class binormal of operator which is defined as $S^{*} S S S^{*}=S S^{*} S^{*} S$.

In [5] Sid Ahmed generalize quasi normal operator to $n$-power quasi normal operator. In this paper we define a new class of operators on Hilbert space as $S^{q}\left(S^{*} S S S^{*}\right)=\left(S S^{*} S^{*} S\right) S^{q}$ called q-power quasi binormal operator and study some properties of it.

\section{Main Results}

Definition 1.1. Let $S$ be bounded operator. Then $S$ is called q-power quasi binormal operator if and only if $S^{q}\left(S^{*} S S S^{*}\right)=\left(S S^{*} S^{*} S\right) S^{q}$, where $q$ is a nonnegative integer.

Proposition 1.2. If $S$ is a self adjoint and q-power quasi binormal operator, then $S^{*}$ is a q-power quasi binormal operator.

Proof. Since $S$ is $q$-power quasi binormal operator, $S^{q}\left(S^{*} S S S^{*}\right)=\left(S S^{*} S^{*} S\right) S^{q}$.

Received: December 24, 2021; Accepted: February 12, 2022

2010 Mathematics Subject Classification: 47B20.

Keywords and phrases: normal operator, quasi normal operator, binormal operator, Hilbert space. 
Let

$\left(S^{*}\right)^{q}\left[\left(\left(S^{*}\right)^{*} S^{*} S^{*}\left(S^{*}\right)^{*}\right)\right]=\left(S^{*}\right)^{q}\left(S S^{*} S^{*} S\right)$, since $S$ is a self adjoint

$$
\begin{aligned}
& =S^{q}\left(S^{*} S S S^{*}\right), \text { since } S \text { is } q \text {-power quasi binormal } \\
& =\left(S S^{*} S^{*} S\right) S^{q}, \text { since } S \text { is a self adjoint } \\
& =\left(S^{*}\left(S^{*}\right)^{*}\left(S^{*}\right)^{*} S^{*}\right)\left(S^{*}\right)^{q} .
\end{aligned}
$$

Hence, $S^{*}$ is $q$-power quasi binormal operator.

Proposition 1.3. If $S$ is a q-power quasi binormal operator, and if $S^{-1}$ exist, then $S^{-1}$ is a q-power quasi binormal operator.

Proof. Since $S$ is $q$-power quasi binormal operator, $S^{q}\left(S^{*} S S S^{*}\right)=\left(S S^{*} S^{*} S\right) S^{q}$.

Let

$$
\begin{aligned}
\left.\left(S^{-1}\right)^{q}\left[\left(S^{-1}\right)^{*} S^{-1} S^{-1}\left(S^{-1}\right)^{*}\right)\right] & \left.=\left(S^{q}\right)^{-1}\left[\left(S^{*}\right)^{-1} S^{-1} S^{-1}\left(S^{*}\right)^{-1}\right)\right] \\
& \left.=\left(S^{q}\right)^{-1}\left[\left(S S^{*}\right)^{-1}\left(S^{*} S\right)^{-1}\right)\right] \\
& =\left(S^{q}\right)^{-1}\left[\left(S^{*} S\right)\left(S S^{*}\right)\right]^{-1} \\
& =\left[\left[\left(S^{*} S S S^{*}\right)\right] S^{q}\right]^{-1}, \text { since } S \text { is binormal } \\
& =\left[\left[\left(S S^{*} S^{*} S\right)\right] S^{q}\right]^{-1}, \\
& \text { since } S \text { is a q-power quasi binormal, } \\
& =\left[S^{q}\left(S^{*} S S S^{*}\right)\right]^{-1}, \text { since } S \text { is binormal } \\
& =\left[S^{q}\left(S S^{*} S^{*} S\right)\right]^{-1} \\
& =\left[\left(S S^{*} S^{*} S\right)\right]^{-1}\left(S^{q}\right)^{-1} \\
& =\left[\left(S^{*} S\right)^{-1}\left(S S^{*}\right)^{-1}\right]\left(S^{q}\right)^{-1} \\
& =\left[S^{-1}\left(S^{*}\right)^{-1}\left(S^{*}\right)^{-1} S^{-1}\right]\left(S^{q}\right)^{-1} \\
& =\left[S^{-1}\left(S^{-1}\right)^{*}\left(S^{-1}\right)^{*} S^{-1}\right]\left(S^{-1}\right)^{q} .
\end{aligned}
$$

Hence, $S^{-1}$ is $q$-power quasi binormal operator.

Definition 1.4 [4]. If $A, B$ are bounded operator on Hilbert space $H$. Then $A, B$ are unitary equivalent if there is an isomorphism $U: H \rightarrow H$ such that $B=U A U^{*}$. 
Proposition 1.5. If $S$ is q-power quasi binormal operator and if $R \in B(H)$ is unitary equivalent to $S$, then $R$ is q-power quasi binormal operator.

Proof. Since $R$ is unitary equivalent to $S, R=U S U^{*},\left(U S U^{*}\right)^{n}=U S^{n} U^{*}$ and since $S$ is $q$-power quasi binormal operator, $S^{q}\left(S^{*} S S S^{*}\right)=\left(S S^{*} S^{*} S\right) S^{q}$.

Let

$$
\begin{aligned}
R^{q}\left(R^{*} R R R^{*}\right) & =\left(U S U^{*}\right)^{q}\left[\left(U S U^{*}\right)^{*}\left(U S U^{*}\right)\left(U S U^{*}\right)\left(U S U^{*}\right)^{*}\right] \\
& =\left(U S^{q} U^{*}\right) \quad\left[\left(U S^{*} U^{*}\right) \quad\left(U S U^{*}\right)\left(U S U^{*}\right)\left(U S^{*} U^{*}\right)\right] \\
& =U\left[S^{q}\left(S^{*} S S S^{*}\right)\right] U^{*}, \text { since } S \text { is q-power quasi binormal operator } \\
& =U\left[\left(S S^{*} S^{*} S\right) S^{q}\right] U^{*} \\
& =\left[\left(U S U^{*}\right)\left(U S^{*} U^{*}\right)\left(U S^{*} U^{*}\right)\left(U S U^{*}\right)\right]\left(U S^{q} U^{*}\right)=\left(R R^{*} R^{*} R\right) R^{q} .
\end{aligned}
$$

Hence $R$ is $q$-power quasi binormal operator.

Theorem 1.6. The set of all q-power quasi binormal operators on $H$ is a closed subset of $B(H)$ under scalar multiplication.

Proof. Let

$$
\begin{array}{r}
M(H)=\{S \in B(H): S \text { is } q \text {-power quasi binormal operator on } H \\
\text { for some nonnegative integer } q\}
\end{array}
$$

Let $S \in W(H)$, then we have $S$ is $q$-power quasi binormal operator and thus $S^{q}\left(S^{*} S S S^{*}\right)=\left(S S^{*} S^{*} S\right) S^{q}$.

Let $\theta$ be a scalar, hence

$$
\begin{aligned}
(\theta S)^{q}\left[(\theta S)^{*}(\theta S)(\theta S)(\theta S)^{*}\right] & \left.=\theta^{q} S^{q}\left[\bar{\theta} S^{*}(\theta S)(\theta S)\right) \bar{\theta} S^{*}\right] \\
& =\theta^{q} \bar{\theta} \theta \theta \bar{\theta}\left[S^{q}\left(S^{*} S S S^{*}\right)\right] \\
& =\theta^{q} \bar{\theta} \theta \theta \bar{\theta}\left[\left(S S^{*} S^{*} S\right) S^{q}\right] \\
& =\left[(\theta S)(\theta S)^{*}(\theta S)^{*}(\theta S)\right](\theta S)^{q}
\end{aligned}
$$

Thus $\theta S \in M(H)$.

Let $S_{k}$ be a sequence in $M(H)$ and converge to $S$, then we can get that 


$$
\begin{aligned}
& \left\|S^{q}\left(S^{*} S S S^{*}\right)-\left(S S^{*} S^{*} S\right) S^{q}\right\| \\
= & \left\|S^{q}\left(S^{*} S S S^{*}\right)-S_{k}^{q}\left(S_{k}^{*} S_{k} S_{k} S_{k}^{*}\right)+\left(S_{k} S_{k}^{*} S_{k}^{*} S_{k}\right) S_{k}^{q}-\left(S S^{*} S^{*} S\right) S^{q}\right\| \\
\leq & \left\|S^{q}\left(S^{*} S S S^{*}\right)-S_{k}^{q}\left(S_{k}^{*} S_{k} S_{k} S_{k}^{*}\right)\right\|+\left\|\left(S_{k} S_{k}^{*} S_{k}^{*} S_{k}\right) S_{k}^{q}-\left(S S^{*} S^{*} S\right) S^{q}\right\| \rightarrow 0 \text { as } k \rightarrow \infty .
\end{aligned}
$$

Hence, $S^{q}\left(S^{*} S S S^{*}\right)=\left(S S^{*} S^{*} S\right) S^{q}$.

Therefore $S \in M(H)$. Then, $M(H)$ is closed subset.

Theorem 1.7. If $T$ and $S$ are normal, q-power quasi binormal operators on $H$, and let $S$ commute with $T$, then (ST) is q-power quasi binormal operator on $H$.

Proof. Since $T$ and $S$ are $q$-power quasi binormal operators $S^{q}\left(S^{*} S S S^{*}\right)=$ $\left(S S^{*} S^{*} S\right) S^{q}$ and $T^{q}\left(T^{*} T T T^{*}\right)=\left(T T^{*} T^{*} T\right) T^{q}$,

$$
\begin{aligned}
(S T)^{q}\left((S T)^{*}(S T)(S T)(S T)^{*}\right) & =\left(T^{q} S^{q}\right)\left[\left(T^{*} S^{*}\right)(S T)(S T)\left(T^{*} S^{*}\right)\right] \\
& =\left(T^{q} S^{q}\right)\left[T^{*} S^{*} S T S T T^{*} S^{*}\right] \\
& =\left(T^{q} S^{q}\right)\left[T^{*} S S^{*} T S T^{*} T S^{*}\right] \\
& =\left(T^{q} S^{q}\right)\left[S T^{*} S^{*} T S T^{*} S^{*} T\right] \\
& =\left(T^{q} S^{q}\right)\left[S T^{*} T S^{*} T^{*} S S^{*} T\right] \\
& =\left(T^{q} S^{q}\right)\left[S T T^{*} S^{*} T^{*} S^{*} S T\right] \\
& =T^{q} S S^{q} T T^{*} S^{*} T^{*} S^{*} S T \\
& =S T^{q} S^{q} T T^{*} S^{*} T^{*} S^{*} S T \\
& =S T^{q} T S^{q} T^{*} S^{*} T^{*} S^{*} S T \\
& =S T T^{q} S^{q} T^{*} S^{*} T^{*} S^{*} S T \\
& \vdots \\
& =\left[(S T)\left(T^{*} S^{*}\right)\left(T^{*} S^{*}\right)(S T)\right]\left(T^{q} S^{q}\right) \\
& =\left((S T)(S T)^{*}(S T)^{*}(S T)\right)(S T)^{q}
\end{aligned}
$$

Then (ST) is $q$-power quasi binormal operator.

Theorem 1.8. Let $T_{1}, T_{2}, \ldots, T_{k}$ are q-power quasi binormal operators on $H$. Then the direct sum $\left(T_{1} \oplus T_{2} \oplus \ldots \oplus T_{k}\right)$ is q-power quasi binormal operator on $H$.

Proof. Since every operator of $T_{1}, T_{2}, \ldots, T_{k}$ is $q$-power quasi binormal, 


$$
\begin{aligned}
& T_{i}^{q}\left(T_{i}^{*} T_{i} T_{i} S^{*}\right) \\
= & \left(T_{i} T_{i}^{*} T_{i}^{*} T_{i}\right) T_{i}^{q} \text { for all } i=1,2, \ldots, k \\
= & \left(T_{1} \oplus T_{2} \oplus \ldots \oplus T_{k}\right)^{q}\left[\left(T_{1} \oplus T_{2} \oplus \ldots \oplus T_{k}\right)^{*}\left(T_{1} \oplus T_{2} \oplus \ldots \oplus T_{k}\right)\right. \\
& \left.\quad \times\left(T_{1} \oplus T_{2} \oplus \ldots \oplus T_{k}\right)\left(T_{1} \oplus T_{2} \oplus \ldots \oplus T_{k}\right)^{*}\right] \\
= & \left(T_{1}{ }^{q} \oplus T_{2}{ }^{q} \oplus \ldots \oplus T_{k}{ }^{q}\right)\left[\left(T_{1}{ }^{*} \oplus T_{2}{ }^{*} \oplus \ldots \oplus T_{k}{ }^{*}\right)\left(T_{1} \oplus T_{2} \oplus \ldots \oplus T_{k}\right)\right. \\
& \left.\quad \times\left(T_{1} \oplus T_{2} \oplus \ldots \oplus T_{k}\right)\left(T_{1}{ }^{*} \oplus T_{2}{ }^{*} \oplus \ldots \oplus T_{k}{ }^{*}\right)\right] \\
= & T_{1}{ }^{q}\left(T_{1}{ }^{*} T_{1} T_{1} T_{1}{ }^{*}\right) \oplus T_{2}{ }^{q}\left(T_{2}{ }^{*} T_{2} T_{2} T_{2}{ }^{*}\right) \oplus \ldots \oplus T_{k}{ }^{q}\left(T_{k}{ }^{*} T_{k} T_{k} T_{k}{ }^{*}\right) \\
= & \left(T_{1} T_{1}{ }^{*} T_{1}{ }^{*} T_{1}\right) T_{1}{ }^{q} \oplus\left(T_{2} T_{2}{ }^{*} T_{2}{ }^{*} T_{2}\right) T_{2}{ }^{q} \oplus \ldots \oplus\left(T_{k} T_{k}{ }^{*} T_{k}{ }^{*} T_{k}\right) T_{k}{ }^{q} \\
= & {\left[\left(T_{1} \oplus T_{2} \oplus \ldots \oplus T_{k}\right)\left(T_{1} \oplus T_{2} \oplus \ldots \oplus T_{k}\right)^{*}\left(T_{1} \oplus T_{2} \oplus \ldots \oplus T_{k}\right)^{*}\left(T_{1} \oplus T_{2} \oplus \ldots \oplus T_{k}\right)\right] } \\
& \quad \times\left(T_{1} \oplus T_{2} \oplus \ldots \oplus T_{k}\right)^{q}
\end{aligned}
$$

Thus, $\left(T_{1} \oplus T_{2} \oplus \ldots \oplus T_{k}\right)$ is $q$-power quasi binormal operator on $H$.

Theorem 1.9. Let $T_{1}, T_{2}, \ldots, T_{k}$ are q-power quasi binormal operators on $H$. Then the tenser product $\left(T_{1} \otimes T_{2} \otimes \ldots \otimes T_{k}\right)$ is q-power quasi binormal operator on $H$.

Proof. Since every operator of $S_{1}, S_{2}, \ldots, S_{k}$ is a $q$-power quasi binormal,

$$
\begin{aligned}
& T_{i}^{q}\left(T_{i}^{*} T_{i} T_{i} S^{*}\right) \\
= & \left(T_{i} T_{i}^{*} T_{i}^{*} T_{i}\right) T_{i}^{q} \text { for all } i=1,2, \ldots, k \\
= & \left(T_{1} \otimes T_{2} \otimes \ldots \otimes T_{k}\right)^{q}\left[\left(T_{1} \otimes T_{2} \otimes \ldots \otimes T_{k}\right)^{*}\left(T_{1} \otimes T_{2} \otimes \ldots \otimes T_{k}\right)\right. \\
& \left.\quad \times\left(T_{1} \otimes T_{2} \otimes \ldots \otimes T_{k}\right)\left(T_{1} \otimes T_{2} \otimes \ldots \otimes T_{k}\right)^{*}\right]\left(x_{1} \otimes x_{2} \otimes \ldots \otimes x_{\mathbf{k}}\right) \\
= & \left(T_{1}{ }^{q} \otimes T_{2}{ }^{q} \otimes \ldots \otimes T_{k}{ }^{q}\right)\left[\left(T_{1}{ }^{*} \otimes T_{2}{ }^{*} \otimes \ldots \otimes T_{k}{ }^{*}\right)\left(T_{1} \otimes T_{2} \otimes \ldots \otimes T_{k}\right)\right.
\end{aligned}
$$

Thus $\left(T_{1} \otimes T_{2} \otimes \ldots \otimes T_{k}\right)$ is $q$-power quasi binormal operator. 


\section{References}

[1] A. Brown, On a class of operators, Proc. Amer. Math. Soc. 4 (1953), 723-728. https://doi.org/10.1090/S0002-9939-1953-0059483-2

[2] S. K. Berberian, Introduction to Hilbert Space, 2nd ed., Chelsea Publishing Co., New York, 1976.

[3] Stephen L. Campbell, Linear operators for which $T^{*} T$ and $T T^{*}$ commute, Proc. Amer. Math. Soc. 34 (1972), 177-180. https://doi.org/10.2307/2037922

[4] J. B. Conway, A course in functional analysis, Graduate Texts in Mathematics, 96, Springer-Verlag, New York, 1985. https://doi.org/10.1007/978-1-4757-3828-5

[5] Ould Ahmed Mahmoud Sid Ahmed, On the class of $n$-power quasi-normal operators on Hilbert space, Bull. Math. Anal. Appl. 3(2) (2011), 213-228.

This is an open access article distributed under the terms of the Creative Commons Attribution License (http://creativecommons.org/licenses/by/4.0/), which permits unrestricted, use, distribution and reproduction in any medium, or format for any purpose, even commercially provided the work is properly cited. 